\title{
Writing and erasing of temporal cavity solitons by direct phase modulation of the cavity driving field
}

\author{
Jae K. Jang, ${ }^{*}$ Miro Erkintalo, Stuart G. Murdoch, and Stéphane Coen \\ Dodd-Walls Centre for Photonic and Quantum Technologies, \\ and Physics Department, The University of Auckland, \\ Private Bag 92019, Auckland 1142, New Zealand \\ *Corresponding author: jake.jang.ur.mate@gmail.com
}

\begin{abstract}
Temporal cavity solitons (CSs) are persisting pulses of light that can manifest themselves in continuously driven passive resonators, such as macroscopic fiber ring cavities and monolithic microresonators. Experiments so far have demonstrated two techniques for their excitation, yet both possess drawbacks in the form of system complexity or lack of control over soliton positioning. Here we experimentally demonstrate a new CS writing scheme that alleviates these deficiencies. Specifically, we show that temporal CSs can be excited at arbitrary positions through direct phase modulation of the cavity driving field, and that this technique also allows existing CSs to be selectively erased. Our results constitute the first experimental demonstration of temporal cavity soliton excitation via direct phase modulation, as well as their selective erasure (by any means). These advances reduce the complexity of CS excitation and could lead to controlled pulse generation in monolithic microresonators.
\end{abstract}

Cavity solitons (CSs) are solitary waves that can persist in driven nonlinear passive resonators [1]. Their tendency to broaden is balanced by nonlinear self-focusing, and all energy they lose is replenished by the coherent field driving the cavity. Several of them can simultaneously co-exist, and they can be independently excited, erased and manipulated [2 [5]. These properties have led to CSs being identified as promising candidates for bits in alloptical buffers and processing units [ $[\underline{8}]$.

Historically the focus has been on spatial CSs, i.e., non-diffracting localized beams of light trapped in planar cavities 2 4]. More recently experiments performed in optical fiber loops [5, 8 10] have stimulated interest in their temporal counterparts: recirculating non-dispersive pulses of light 7]. Such temporal CSs do not suffer from material defects that hinder the performance of spatial cavities [1], although somewhat analogous impairments can arise due to electrostriction-mediated interactions [9]. These interactions can however be overcome by phase modulating the continuous wave $(\mathrm{cw})$ laser driving the cavity; the CSs are then trapped to the peaks of the ensuing intracavity phase profile 2, 5]. In parallel with investigations in macroscopic fiber cavities, temporal CSs have also attracted attention in the context of monolithic microresonators [12, 13]. In these devices, CSs can under certain conditions correspond to the temporal structures underlying broadband "Kerr" frequency combs [14, 15], suggesting applications in metrology and high repetition rate pulse train generation.

Excitation of CSs requires that the resonator $\mathrm{cw}$ steady-state is suitably perturbed [6, 16 18]. For temporal CSs two techniques have been demonstrated. Experiments in fiber resonators have used an incoherent "writing" scheme [5, 8 10], where an optical "addressing" pulse perturbs the cw steady-state via nonlinear cross-phase modulation (XPM). In microresonators, sta- ble soliton states have been reached by carefully tuning the frequency of the cavity driving field [12, 13]. Both methods yield CSs, but also possess drawbacks: optical addressing increases the system complexity [8], whilst adjusting the driving laser frequency provides limited control over how many CSs are excited and at what temporal positions [15]. Moreover, neither of these techniques can easily be adapted to achieve controlled erasure of existing CSs. XPM has been numerically proposed for this purpose [8], but difficulties in synchronization have prevented experimental realization. This represents a major shortcoming, since the potential to be independently erased is widely regarded as a defining characteristic of CSs, underpinning many of their proposed functionalities [4, 16, 17].

Here, we implement another method for temporal CS excitation that alleviates these deficiencies. Our technique relies on the phase modulation that is typically applied to the cavity driving field to trap CSs into specific time-slots [5]. By applying local boosts in the phase modulation, we are able to write CSs into corresponding empty slots. Significantly, similar boosts also allow us to demonstrate selective erasure of CSs already trapped in the cavity. This technique minimizes the complexity of CS systems, since the same components enable writing, trapping, and erasure. It could also represent a step towards controlled pulse generation in optical microresonators [19].

The ability to switch dispersive $\mathrm{cw}$ bistable devices through appropriate changes of the phase of the driving field was first proposed by Hopf et al. 20], and analyzed theoretically in the context of CSs by McDonald and Firth [18]. Phase modulation has also been used recently to excite localized topological phase structures in a laser with external forcing and feedback 21]. For a better understanding of our experiment, we first illus- 
trate the phase-induced switching dynamics of temporal CSs by means of numerical simulations. To this end, we model the evolution of the field envelope $E(t, \tau)$ inside a high-finesse fiber resonator using the well-known meanfield equation [7, 8, 14]:

$$
\begin{array}{r}
t_{R} \frac{\partial E}{\partial t}=\left(-\alpha+i \gamma L|E|^{2}-i \delta_{0}-i \frac{\beta_{2} L}{2} \frac{\partial^{2}}{\partial \tau^{2}}\right) E(t, \tau) \\
+\sqrt{\theta} S(t, \tau)
\end{array}
$$

Here, $t$ is a slow time variable that describes evolution over consecutive roundtrips and $\tau$ is a fast time that describes the temporal profile of the field envelope. $t_{\mathrm{R}}$ is the cavity roundtrip time, $\alpha$ is half the fraction of power lost per roundtrip, $\gamma$ is the nonlinearity coefficient, $L$ is the cavity length and $\beta_{2}$ is the group-velocity dispersion coefficient. The parameter $\delta_{0}$ describes the phase detuning of the driving field $S(t, \tau)$ from the nearest cavity resonance and $\theta$ is the power transmission coefficient used to couple light into the resonator. The driving field is cw with power $P_{\text {in }}$, periodically phasemodulated with a one-bit pattern synchronized to the cavity free-spectral range, $S(t, \tau)=P_{\text {in }}^{1 / 2} \exp [i \phi(t, \tau)]$, where $\phi(t, \tau)=A(t) \exp \left(-\tau^{2} / \tau_{0}^{2}\right)$. Our simulations use parameters similar to the experiments that follow (see caption of Fig. 10,

Figure 1 illustrates how CS writing and erasing can be achieved by dynamically controlling the phase modulation amplitude $A(t)$. Starting with a cold cavity, we turn on the driving field with a weak Gaussian [70 ps fullwidth at half maximum (FWHM)] phase modulation, $A=0.52 \mathrm{rad}$, which mimics that used in our experiments to overcome soliton interactions [5, 9]. As can be seen, this weak phase modulation alone does not lead to CS excitation. Instead, after some tens of roundtrips the field converges to a cw steady-state. After 100 roundtrips we abruptly boost the phase modulation amplitude to $A=2.17 \mathrm{rad}$ for 10 roundtrips. This leads to a broad peak in the optical intensity which evolves and settles into a $2.6 \mathrm{ps}$ wide (FWHM) CS in about 100 roundtrips. The CS then persists until we again boost the phase modulation amplitude to 2.17 rad for 15 roundtrips, which erases the CS after a brief transient.

The ability to write and erase using phase modulation can be understood by noting that an abrupt step in the driving phase is equivalent to rotating the intracavity field in complex phase-space around the origin [20]. Writing (erasing) occurs if the sequence of rotations, combined with the intervening nonlinear evolution, is such that the field falls within the basin of attraction of a single CS (lower-state cw). This of course implies that not all phase operations lead to switching, as is illustrated for CS writing in Fig. 2. Here we plot the simulated evolution of the amplitude $E(t, \tau=0)$ in the complex plane (with phase calculated relative to that of the driving field at $\tau=0$ ), starting from the cw solution, for three dif-

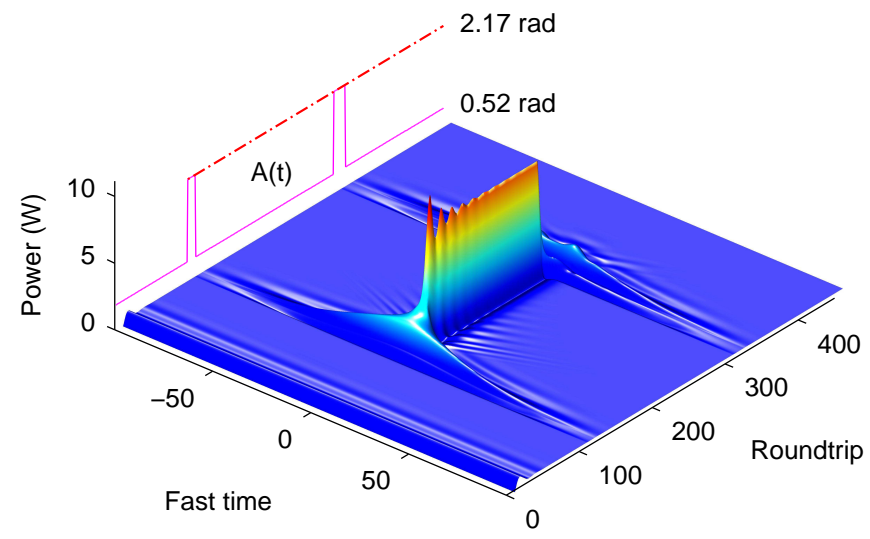

Figure 1. Numerical results of CS writing and erasing by means of abrupt changes in the phase of the cavity driving field. The phase modulation amplitude $A(t)$ is shown as the curve on the left. The numerical parameters are similar to the experiments that follow: $P_{\mathrm{in}}=908 \mathrm{~mW}, t_{\mathrm{R}}=0.48 \mu \mathrm{s}$, $L=100 \mathrm{~m}, \theta=0.1, \alpha=0.146, \gamma=1.2 \mathrm{~W}^{-1} \mathrm{~km}^{-1}, \beta_{2}=$ $-21.4 \mathrm{ps}^{2} / \mathrm{km}, \delta_{0}=0.4426 \mathrm{rad}$, and $\tau_{0}=43 \mathrm{ps}$.

ferent phase sequences. The blue curve corresponds to the successful writing procedure in Fig. 1] whereby the phase modulation amplitude is first boosted to $2.17 \mathrm{rad}$ (the corresponding rotation is labeled as step 1) and then reduced back to 0.52 rad after 10 roundtrips (step 2). As can be seen, the field eventually spirals to the stable CS solution (this spiraling represents the transient oscillations seen in Fig. 10. In contrast, if the phase modulation amplitude during the 10-roundtrip boost is slightly lower (2.00 rad, red curve), no writing takes place; the field remains in the basin of attraction of the original cw solution. Similar behavior is observed when the modulation amplitude is only boosted but never returned to its original value, and the green curve in Fig. 2 shows a trajectory for this case. We note, however, that these dynamics are particular to our choice of parameters, and for others, writing can be achieved even with a single

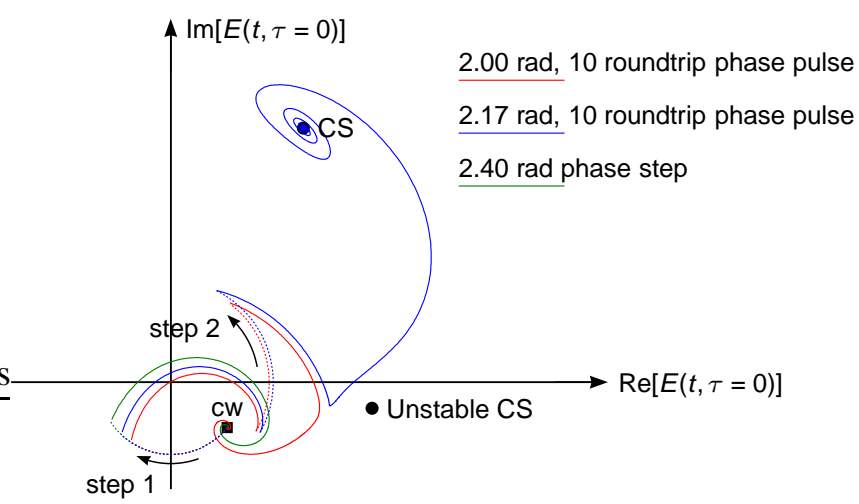

Figure 2. Simulated phase-space dynamics during attempts of CS writing with three different phase-modulation sequences. 
phase-step [18]. In fact, the precise dynamics depends quite sensitively on the parameters involved. This may be linked to the metastable nature of the unstable CS solution (which exists for the same parameters [8]), and to which the emerging soliton field is initially attracted to (see blue curve in Fig. 1), as well as to the associated non-critical slowing [18]. We find that, for given parameters, some trial and error is required to determine phase sequences suitable for writing and erasing. Fortunately this task is quite effortless; whilst the precise dynamics may somewhat differ, switching nevertheless occurs over a wide range of conditions. We must remark that CS switching can also be realized with abrupt changes to other parameters, even with a static phase profile. Such changes can be induced by, e.g., mechanically perturbing the cavity.

To experimentally demonstrate CS writing and erasing, we use the set-up schematically illustrated in Fig. 3 . The optical cavity is similar to that used in [5, 9], consisting of 100 meters of single-mode fiber (SMF) closed on itself with a $90 / 10$ fiber coupler. The cavity is driven with a narrow linewidth $\mathrm{cw}$ laser at $1550 \mathrm{~nm}$ whose output is phase-modulated with an electro-optic modulator (EOM) and then amplified with an erbium-doped fiber amplifier (EDFA). An electronic feedback control loop is used to actively lock the laser frequency near a cavity resonance. The cavity also hosts a fiber isolator, used to suppress stimulated Brillouin scattering, as well as a 99/1 tap-coupler, through which the intracavity dynamics are monitored with a $12 \mathrm{GHz}$ real-time oscilloscope. Before detection, the cavity output is filtered using a narrow (0.6 nm width) bandpass filter (BPF) centered at $1551 \mathrm{~nm}$. This improves the signal-to-noise ratio by removing the $\mathrm{cw}$ component [8].

The abrupt phase changes that enable switching are achieved by manipulating the electronic signal that drives the EOM [see Fig. 3(b)]. Two programmable pattern generators, capable of producing approximately $70 \mathrm{ps}$

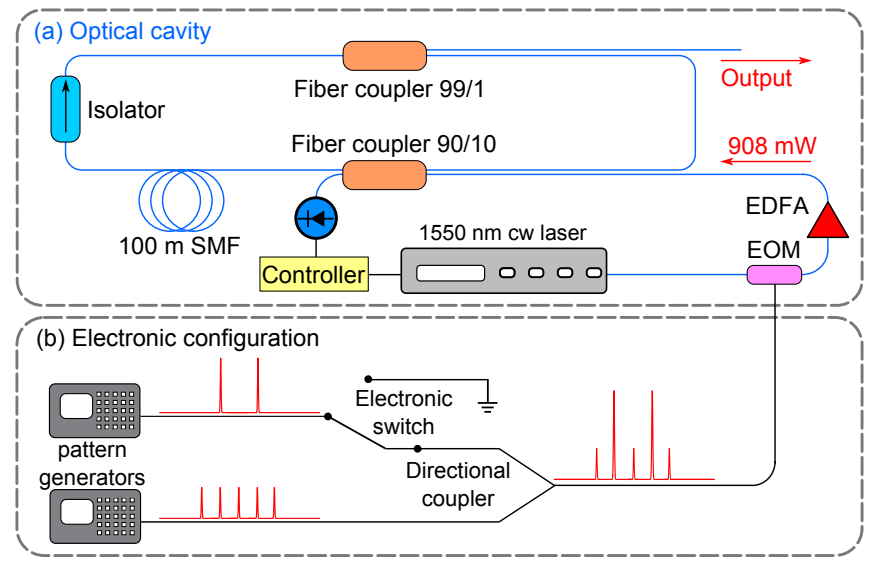

Figure 3. Schematic illustration of the (a) optical and (b) electronic segments of the experimental setup.
FWHM electronic pulses, are synchronised by a single external clock, such that the repetition rate of their output patterns coincide with the cavity free-spectral range. One of them [top in Fig. 3(b)] is set to selectively produce pulses whose amplitude is $\sim 3$ times larger than those from the other. The low-amplitude pattern is fed to the EOM at all time; this gives rise to an intracavity phase profile that allows CSs to be trapped [5], but not written or erased. In contrast, the high-amplitude pattern is controlled by an electronically gated switch. When the switch is activated, the outputs of both pattern generators are combined for about 10 roundtrips. In this way the amplitudes of those electronic pulses that are contained in the high-amplitude pattern can be selectively boosted to $\sim 4$ times their original level.

For a particular experimental demonstration, we set the low-amplitude pattern generator to produce a sequence of 5 electronic pulses separated by 300 ps (the whole pattern repeats once per cavity roundtrip). We then explore complex writing and erasing sequences by selectively boosting some of the amplitudes as described above. Experimental results are shown in Fig. 4. The density map concatenates successive oscilloscope traces recorded at the cavity output, illustrating how the optical intensity inside the resonator is affected by abrupt changes in phase modulation amplitude (the bit sequences on the right indicate which electronic pulses are boosted in amplitude). During the first 5 seconds of measurement no CSs are excited, confirming that the lowamplitude modulation itself is insufficient for this purpose. At $t=5 \mathrm{~s}$ all five electronic pulses are boosted for 10 roundtrips, resulting in the creation of five CSs. After excitation, the CSs remain trapped to the peaks

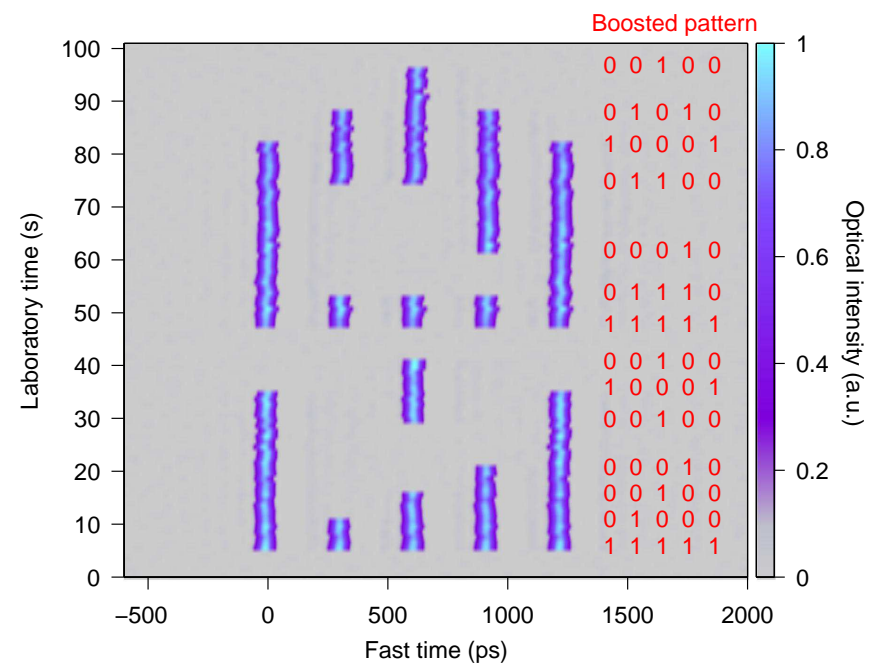

Figure 4. Experimental density map showing successive oscilloscope traces of the optical intensity at the cavity output. All the traces were acquired at 1 frame/s with a $40 \mathrm{GSa} / \mathrm{s}$ real-time oscilloscope. The bit sequences on the right indicate which of the five phase pulses are boosted to achieve writing and erasing. 
of the low-amplitude phase modulation [2, 5]. We then demonstrate erasure of individual CSs, by boosting selected phase pulses already trapping a CS, and highlight the flexibility of the scheme by performing complex simultaneous writing and erasing operations. In this context we remark that we are experimentally able to write and erase using the same operation (same phase amplitude applied over the same number of roundtrips). This should be contrasted with simulations of Fig. 1] where different number of roundtrips were required for writing and erasure. We believe this discrepancy arises due to environmental fluctuations that alter the system parameters during the measurement. This notion is supported by the fact that we do not achieve $100 \%$ fidelity, but instead several attempts are occasionally required for successful switching.

The above experiment demonstrates that direct phase modulation allows temporal CSs to be written and erased. However, the acquisition rate in this measurement was limited to 1 frame/s, which is too slow to capture the switching transients. To gain more insights, we have thus also taken real-time measurements that resolve the roundtrip-to-roundtrip dynamics of the optical intensity at the cavity output together with the electronic signal that drives the EOM. As above, we use the same phase operation for both writing and erasing (except that we use here only one electronic pulse per roundtrip), and Fig. 5(a) shows the corresponding electronic signal. Experimental results for transient writing and erasing dynamics are shown in Figs. 5(b) and (c), respectively. During writing, the field displays ringing similar to that observed during optical excitation [8] and numerical simulations (Fig. 1), and stabilizes in about 100 roundtrips after the moment of addressing. Erasure, on the other hand, appears to take place almost immedi-

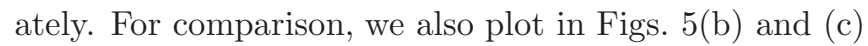
results from numerical simulations (green curves), taking into account the effects of the BPF and the limited bandwidth of our photodetector. The simulations use parameters approximated from experimental measurements (see caption of Fig. 10, yet for erasure we allowed $5 \%$ increase in the cavity detuning $\delta_{0}$ so that both writing and erasure can be achieved with the same experimentally used phase sequence. We believe this is justified given the imperfections in the electronics used to stabilize the laser frequency. Regardless, the numerical results show good qualitative agreement with experiments. In this context, we also note that the writing (erasing) dynamics appear slower (faster) than they truly are since the optical BPF does not capture the broad temporal feature that appears during switching (see Fig. 1).

To conclude, we have experimentally demonstrated writing and erasing of temporal CSs by direct manipulation of the phase profile of the cavity driving field. The demonstrated technique offers a means to simplify the implementation of temporal CS-based all-optical buffers,

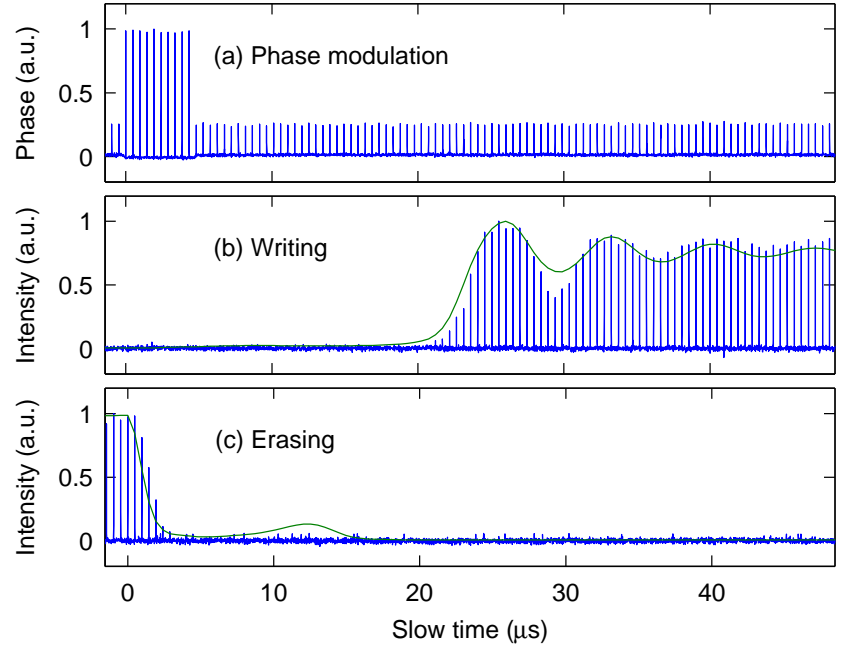

Figure 5. Real-time dynamics of CS writing and erasing. (a) Electronic signal fed to the EOM. (b, c) Experimentally measured roundtrip-to-roundtrip dynamics of (b) writing and (c) erasing. The green curves show results from numerical simulations.

and could enable controlled pulse train generation in monolithic microresonators. In this context, we must emphasize that writing is achieved with phase pulses that have a much longer duration than the CSs (70 ps versus $2.6 \mathrm{ps}$ ). Our work also reports the first experimental demonstration of temporal CS erasure. This highlights that temporal CSs are truly independent entities, and constitutes a step towards their use as fully addressable bits in optical buffers.

[1] T. Ackemann, W. J. Firth, and G. L. Oppo, "Fundamentals and applications of spatial dissipative solitons in photonic devices," Adv. At. Mol. Opt. Phys. 57, 323-421 (2009).

[2] W. J. Firth and A. J. Scroggie, "Optical bullet holes: Robust controllable localized states of a nonlinear cavity," Phys. Rev. Lett. 76, 1623-1626 (1996).

[3] M. Brambilla, L. A. Lugiato, and M. Stefani, "Interaction and control of optical localized structures," Europhys. Lett. 34, 109-114 (1996).

[4] S. Barland, J. R. Tredicce, M. Brambilla, L. A. Lugiato, S. Balle, M. Giudici, T. Maggipinto, L. Spinelli, G. Tissoni, T. Knödl, M. Miller, and R. Jäger, "Cavity solitons as pixels in semiconductor microcavities," Nature 419, 699-702 (2002).

[5] J. K. Jang, M. Erkintalo, S. Coen, and S. G. Murdoch, "Temporal tweezing of light: trapping and manipulation of temporal cavity solitons," arXiv:1410.4836 [nlin.PS, physics.optics] (2014).

[6] G. S. McDonald and W. J. Firth, "Spatial solitarywave optical memory," J. Opt. Soc. Am. B 7, 1328-1335 (1990).

[7] S. Wabnitz, "Suppression of interactions in a phaselocked soliton optical memory," Opt. Lett. 18, 601-603 
(1993).

[8] F. Leo, S. Coen, P. Kockaert, S.-P. Gorza, Ph. Emplit, and M. Haelterman, "Temporal cavity solitons in onedimensional Kerr media as bits in an all-optical buffer," Nature Photon. 4, 471-476 (2010).

[9] J. K. Jang, M. Erkintalo, S. G. Murdoch, and S. Coen, "Ultraweak long-range interactions of solitons observed over astronomical distances," Nature Photon. 7, 657-663 (2013).

[10] J. K. Jang, M. Erkintalo, S. G. Murdoch, and S. Coen, "Observation of dispersive wave emission by temporal cavity solitons," Opt. Lett. 39, 5503-5506 (2014).

[11] E. Caboche, F. Pedaci, P. Genevet, S. Barland, M. Giudici, J. Tredicce, G. Tissoni, and L. A. Lugiato, "Microresonator defects as sources of drifting cavity solitons," Phys. Rev. Lett. 102, 163901 (2009).

[12] T. Herr, V. Brasch, J. D. Jost, C. Y. Wang, N. M. Kondratiev, M. L. Gorodetsky, and T. J. Kippenberg, "Temporal solitons in optical microresonators," Nature Photon. 8, 145-152 (2014).

[13] K. Saha, Y. Okawachi, B. Shim, J. S. Levy, R. Salem, A. R. Johnson, M. A. Foster, M. R. E. Lamont, M. Lipson, and A. L. Gaeta, "Modelocking and femtosecond pulse generation in chip-based frequency combs," Opt. Express 21, 1335-1343 (2013).

[14] S. Coen, H. G. Randle, T. Sylvestre, and M. Erkintalo, "Modeling of octave-spanning Kerr frequency combs using a generalized mean-field Lugiato-Lefever model,"
Opt. Lett. 38, 37-39 (2013).

[15] M. Erkintalo and S. Coen, "Coherence properties of Kerr frequency combs," Opt. Lett. 39, 283-286 (2014).

[16] X. Hachair, L. Furfaro, J. Javaloyes, M. Giudici, S. Balle, J. R. Tredicce, G. Tissoni, L. A. Lugiato, M. Brambilla, and T. Maggipinto, "Cavity-solitons switching in semiconductor microcavities," Phys. Rev. A 72, 013815 (2005).

[17] S. Barbay, Y. Ménesguen, X. Hachair, L. Leroy, I. Sagnes, and R. Kuszelewicz, "Incoherent and coherent writing and erasure of cavity solitons in an optically pumped semiconductor amplifier," Opt. Lett. 31, 1504-1506 (2006).

[18] G. S. McDonald and W. J. Firth, "Switching dynamics of spatial solitary wave pixels," J. Opt. Soc. Am. B 10, 1081-1089 (1993).

[19] H. Taheri, A. A. Eftekhar, K. Wiesenfeld, and A. Adibi, "Soliton formation in whispering-gallery-mode resonators via input phase modulation," arXiv:1409.5943 [nlin.PS, physics:optics] (2014).

[20] F. A. Hopf, P. Meystre, P. D. Drummond, and D. F. Walls, "Anomalous switching in dispersive optical bistability," Opt. Commun. 31, 245-250 (1979).

[21] B. Garbin, J. Javaloyes, G. Tissoni, and S. Barland, "Topological solitons as addressable phase bits in a driven laser," Nature Commun. 6, 5915 (2015). 